\title{
Evaluation of an E-nose system for objective smell assessment of shoe/socks systems by comparison with a sensory panel
}

\author{
$\underline{\text { Stephan Horras }}{ }^{1}$, Alessandra Gaiotto ${ }^{2}$, Maria Mayer ${ }^{3}$, Peter Reimann ${ }^{1}$, Andreas Schütze ${ }^{1}$ \\ ${ }^{1}$ Laboratory for Measurement Technology, Saarland University, Saarbrücken, Germany; \\ ${ }^{2}$ Prüf- und Forschungsinstitut Pirmasens, Pirmasens; ${ }^{3}$ Forschungsinstitut Hohenstein, Boennigheim; \\ Email: s.horras@LMT.uni-saarland.de
}

\begin{abstract}
In this paper we report on the evaluation of an E-nose system for objective evaluation of the smell of sweat in shoe/socking systems by comparison with a human sensory panel. The test system is based on temperature cycled semiconductor gas sensors to achieve good stability for field use of the system without the requirement for frequent recalibration. The ultimate goal is to provide a tool for developing improved shoe/sock systems with optimized materials.

The main approach to achieve this goal is to find a correlation between the assessment of a human sensory panel and the complex sensor response patterns of an E-nose system to appraise the smell of sweat in shoes and socks. Therefore a range of test persons wear shoes and socks under defined ambient conditions in a controlled test environment as well as during everyday use. The evaluation is done by comparison of the data measured by the E-nose system with two independent sensory panels, each consisting of six persons. The human sensory panels evaluate the smell on worn shoes and socks and assess the intensity of the smell of sweat and the olfactory sensation/unpleasantness for each test object. The paper describes the setup of the E-nose system and presents results of a first larger field test showing the identified correlation between our system and the human assessment of the smell of sweat.
\end{abstract}

\section{Motivation}

Suppression or mitigation of body odors is one of the key issues for shoe and clothes manufacturers in Europe to achieve a competitive advantage over low cost suppliers especially from Far East. Specifically the smell of sweat in shoes and socks is a major issue for consumers and manufacturers alike and will eventually be an essential aspect for customer acceptance. Today, only very complex studies with human sensory panels allow an estimation of the impact of different construction characteristics of the shoe/socks system, i.e. the materials used and their combinations. In this respect shoes present a major problem as they usually are not or cannot be washed, so that substances and microbes can accumulate and lead to quite severe smells.

A collaborative project between the lab for measurement technology and two research institutes specialized on shoes and clothing, respectively, aims at developing a mobile test system for objective evaluation of the strength and unpleasantness of the smell of sweat to be used in development of shoes and socks, in quality control and for settling customer complaints. Preliminary studies have shown a correlation between the signals of an electronic nose based on semiconductor sensors and human perception [1]. The key will be to identify characteristic sensor response patterns for correlation with the assessment by a human sensory panel, a typical approach for developing an application specific electronic nose [2].

\section{Experimental methods}

The E-nose system is based on a small test chamber in which the shoe and/or socks are placed for evaluation, Fig. 1 [3]. Gas is pumped from inside the chamber using a probe which can be placed inside the shoe or socks and passes through a sensor chamber with up to eight different semiconductor gas sensors (SGS). To suppress unwanted influence by humidity and interfering gases, the chamber is flushed with pressurized clean air, which is humidified to almost saturation. In addition, the test chamber can be placed in a temperature chamber to suppress the influence of ambient temperature. Temperature and humidity in the test chamber and in the chamber for gas sensors are measured, which will allow a correction of the influence of ambient conditions at a later stage, i.e. by taking the temperature and r.h. values into account for the signal interpretation.

As many electronic noses based on sensor arrays show a lack of stability and therefore require frequent recalibration, we chose to use a system based on temperature cycling which has proven highly stable for the detection of organic substances over several months [4]. Each sensor is controlled by an individual electronics board for temperature cycling and data acquisition [5] and is connected via USB to a laptop computer with graphical user interface based on LabVIEW, NI, for signal evaluation and 




Synthetic Air

Fig. 1: Measurement set-up consisting of the test chamber placed in a temperature chamber, chamber for gas and humidity sensors, sensor electronics and graphical LabVIEW-user interface on a laptop. The test chamber is flushed with synthetic air of variable humidity.

interpretation. Commercial gas sensors [6] that had shown good stability and high selectivity in dynamic operation for other applications were used for these experiments. The temperature cycle chosen for this application has a duration of 40 seconds and covers a temperature range from $240^{\circ} \mathrm{C}$ to $420^{\circ} \mathrm{C}$ by setting three different temperature levels $\left(420^{\circ} \mathrm{C}, 240^{\circ} \mathrm{C}, 330^{\circ} \mathrm{C}\right)$, Fig. 2 .

Each shoe and sock was measured for $20 \mathrm{~min}$. after wearing parallel to the odor assessment by a human sensory panel. Each experiment therefore yields 30 temperature cycles (T-Cycles), which are treated as individual measurements for further interpretation. After data preprocessing (i.e. normalization of the T-cycle data) [7], features are extracted from the cycles forming the basis for multivariate data analysis using standard techniques like principal component analysis (PCA), linear discriminant analysis

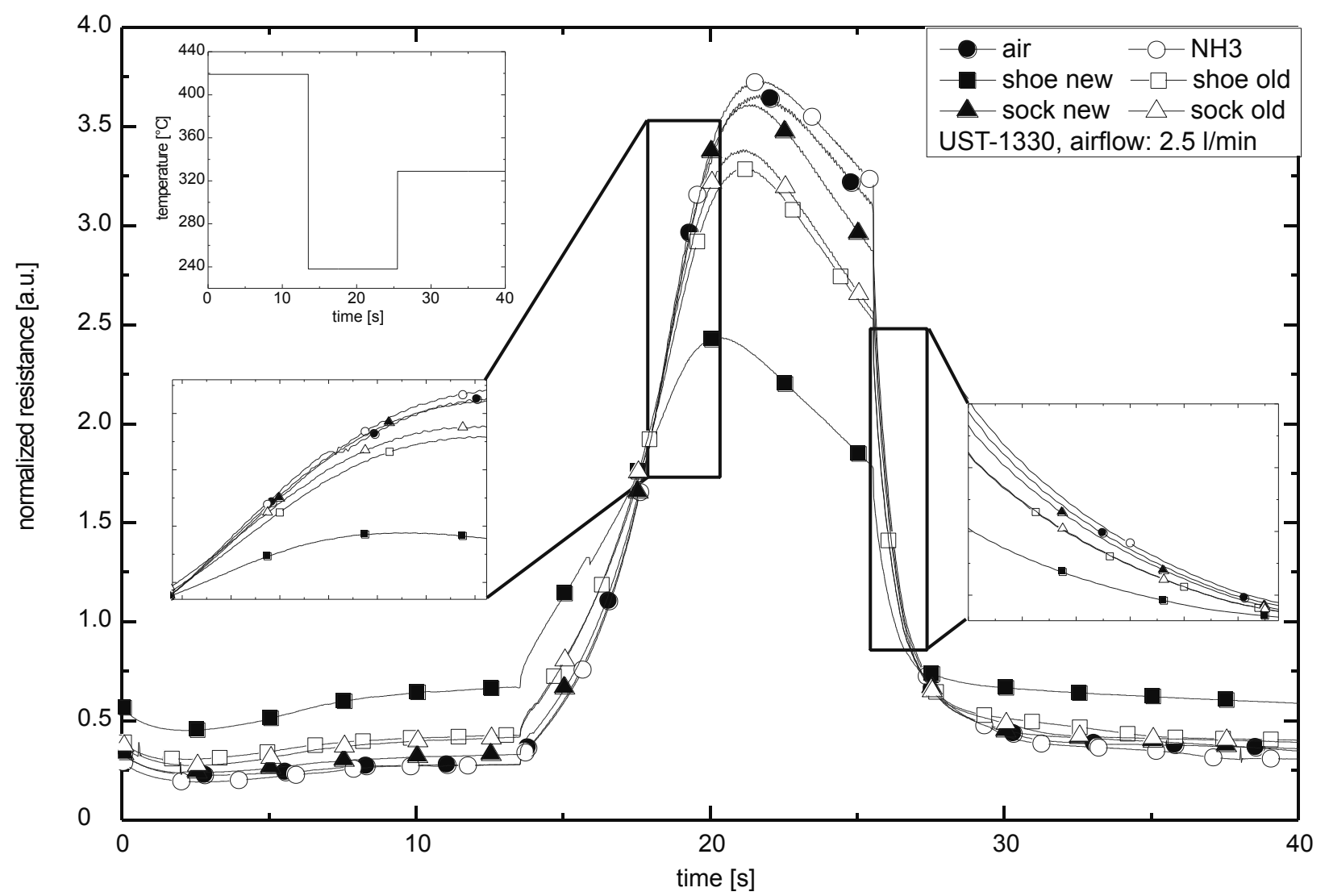

Fig. 2: Response patterns of one semiconductor gas sensor when exposed to air, $\mathrm{NH}_{3}$ (for calibration) and the odors from new and worn socks and shoes, respectively. Inserts show details of the sensor response. Note that the patterns for worn shoes and socks are quite similar to each other, while the patterns for new shoes and socks are distinctively different and also different from each other. 
(LDA) and artificial Neural Networks (ANN) [8]. The patterns show significant differences in the zoomed areas like different slopes, mean values or absolute maxima. We have used the mean values and the slopes during different parts of the T-cycle as these features, which basically describe the shape of the sensor response and have proven valuable before [9]. First tests with the E-nose system had shown that a separation of air, new and worn shoes, respectively, was possible [3]. Furthermore, Fig. 2 shows that patterns of a worn shoe and a worn sock are similar compared to each other and distinctively different from the patterns obtained from a new shoe and sock, respectively. This indicates that the system is suitable for the detection of smell of sweat.

The two project partners Prüf- und Forschungsinstitut Pirmasens (PFI) and Hohenstein Institutes (HI) were responsible for a large range of tests with commercially manufactured shoes and socks and also for the assessment of the smell with human sensory panels. In a first series of tests 96 experiments were made with four different shoes and three different socks both under controlled and repeatable laboratory conditions as well as during everyday use. These tests form the basis for the correlation between the human nose and our E-nose system. Shoes and socks are worn by test persons and afterwards assessed by the human sensory panel and measured with the E-nose system.

Test persons at PFI were required to run or walk for one hour on a treadmill in a climate controlled chamber to sweat the shoes/socks under defined and reproducible ambient conditions. Test persons at $\mathrm{HI}$ on the other hand were wearing the shoes/socks for four hours under everyday conditions. The shoes and socks as well as the tested combinations of shoes and socks were the same for both partners.

The sensory panels at PFI and $\mathrm{HI}$ consisted of 6 persons each, 3 men and 3 women. As a prior qualification check all persons were tested with 12 smell sticks with different everyday odors and had to assign at least 10 of them to the correct odor group to be eligible for the sensory panel. At each institute a total of 10 test persons were available so that a sufficient panel was ensured for all tests. The sensory panels assess the smell of both shoe and socks individually in the categories overall olfactory sensation (pleasant to unpleasant on a scale from -2 to 2) and intensity of the smell of sweat (on a scale from 1 to 5). Furthermore, a detailed assessment of the categories sweet, sour and overpowering odor is done on a scale from 1 to 4 each. In this paper we concentrate only on the sweat odor intensity or score. The assessment of the panels resulted in average grades which were used as reference or nominal values for the correlation with the E-Nose system. Note that most experiments yielded a standard deviation of the odor score of .4 showing that the assessment by the sensory panel is itself not a very exact process.

Both the E-nose measurements and the assessment by the human sensory panels were repeated one day and six days after wearing to determine how the sweat odor of shoes and socks changes over time. Shoes and socks are individually stored in plastic bags during storage to suppress unwanted influences from the outside during that duration.

\section{Results}

Preliminary tests with a standard E-nose system indicated that assessment of the smell of sweat seemed possible [1]. First experiments with our setup had shown that a separation of air, new and worn shoes, respectively, is possible [3]. Furthermore, Fig. 3 shows that an LDA projection based on the Enose measurements of new and worn shoes as well as $\mathrm{NH}_{3}$ as calibration gas (Fig. 3, left) applied to new and worn socks yields a separation of the shoes with a classification in good agreement with the training data (Fig. 3, right). All tests with new socks are projected into the same area as that obtained for new shoes, the majority of the measurements with worn socks is projected into the area for worn shoes with only relatively few misinterpretations in the borderline regions for new and $\mathrm{NH}_{3}$, respectively. This can also be seen from the raw T-cycle data, which are similar for worn shoes and socks and distinctively different from the patterns obtained from a new shoe and sock, respectively, as shown in Fig. 2 [7]. This indicates that the system is suitable for the detection of smell of sweat, because the underlying matrix is of minor importance compared to the sweat odor.

However, this quality is only achieved using LDA as a tool for dimensionality reduction as shown in Fig. 4, comparing PCA and LDA for a small number of experiments. In both cases, the T-cycle data for six experiments with worn shoes were used for the projections. The experiments were selected based on the sweat odor intensity as determined by the human sensory panel: only shoes with an odor score of 1.3 , 2.3 or 3.3 were selected in order to achieve a tight grouping of the experiments with a good separation between the groups with different odor scores. Fig. 4 left shows that the unsupervised PCA yields six groups corresponding to the six different experiments with no correlation to the odor score (experiments with scores of 2.3 are scattered over the whole plot). The LDA projection on the other hand (Fig. 4, right), shows a tight clustering of the experiments with the same odor score and good separation between the groups as desired. The supervised LDA is mathematically optimal for this type of classification or better separation [8]. It is interesting to note, that the separation is basically along the first discriminant function (DF1) and that the odor score correlates to the DF1 value (in this case, a higher odor score leads to a 

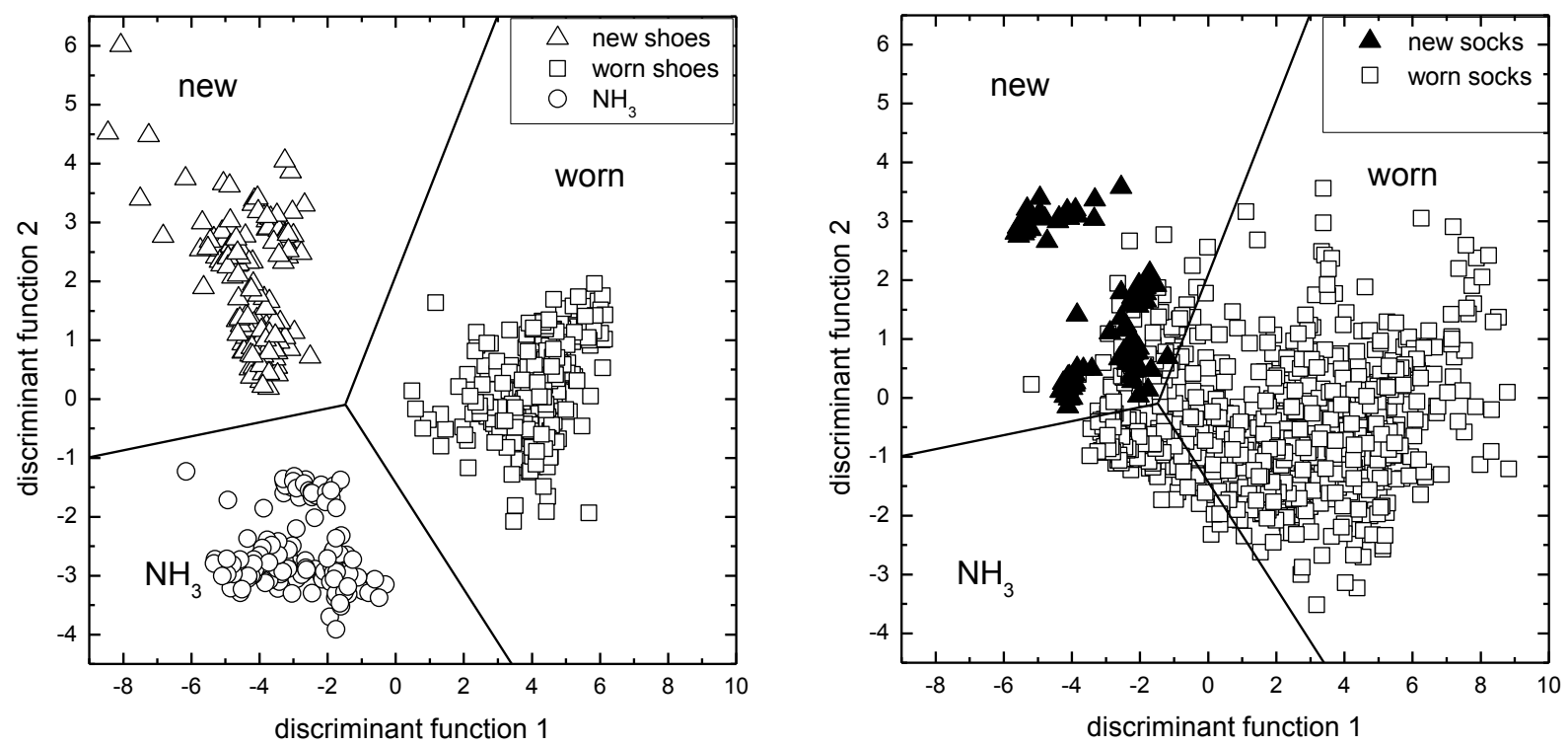

Fig. 3: Left: LDA projection separating the three groups "new shoes", "worn shoes" and $\mathrm{NH}_{3}$ (calibration); right: LDA projection with the LDA coefficients obtained from the shoes $/ \mathrm{NH}_{3}$ data, showing a separation between new and worn (i.e. smelling of sweat) socks based on the same sensor response features. This indicates that the E-nose is capable of identifying the smell of sweat independently of the type of test object.

lower DF1 value). This seems to indicate that the DF1 of a suitable LDA projection can be used to directly predict the odor score by using a linear transfer function. This could already be shown in the past for the determination of a gas concentration based on LDA projections for ozone measurements [10].

The results of the LDA projection with a limited number of test data shown in Fig. 4 suggests that a correlation between the PCA projection along one axis and the odor score can be found thus allowing an easy prediction of the sweat odor intensity using a linear function based on the gas sensor data. This was tested using the data of all 96 experiments performed at $\mathrm{HI}$, i.e. after wearing all four shoes in all combinations with the three different socks and testing shoes and socks separately, also for left and right foot. Fig. 5 shows the result of an LDA projection using the rounded odor score as obtained from the human sensory panel as reference data (left). This time the groups are much less well defined and a
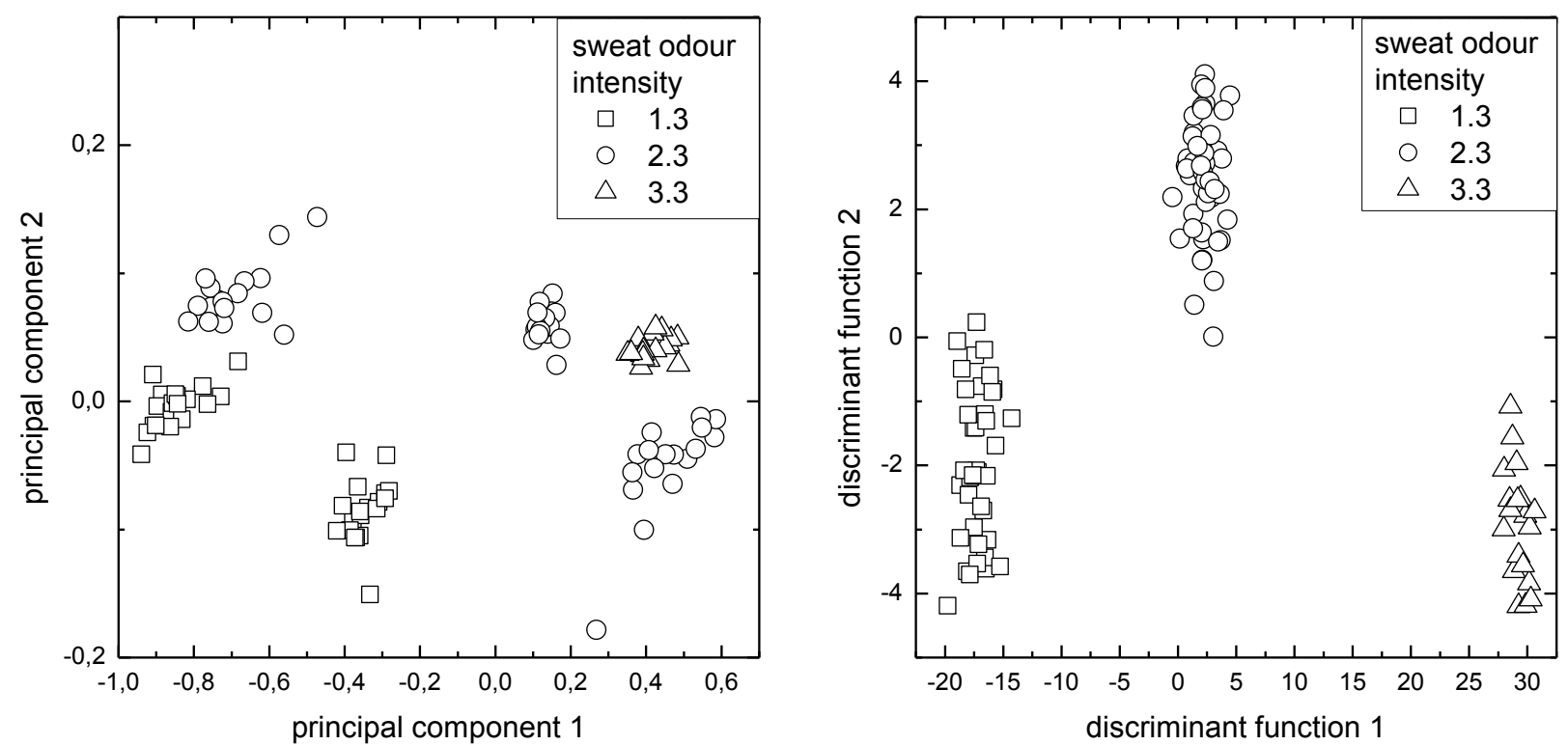

Fig. 4: Comparison of PCA (left) and LDA (right) projections for six different experiments with worn shoes. For this only shoes with an odor intensity score of 1.3, 2.3 or 3.3, respectively, were selected. While the PCA projection shows six scattered groups (one for each shoe) with no correlation to the odor intensity, the LDA projection again shows a clear correlation between the odor intensity and DF1 allowing a classification of the odor intensity as evaluated by the human sensory panel. 

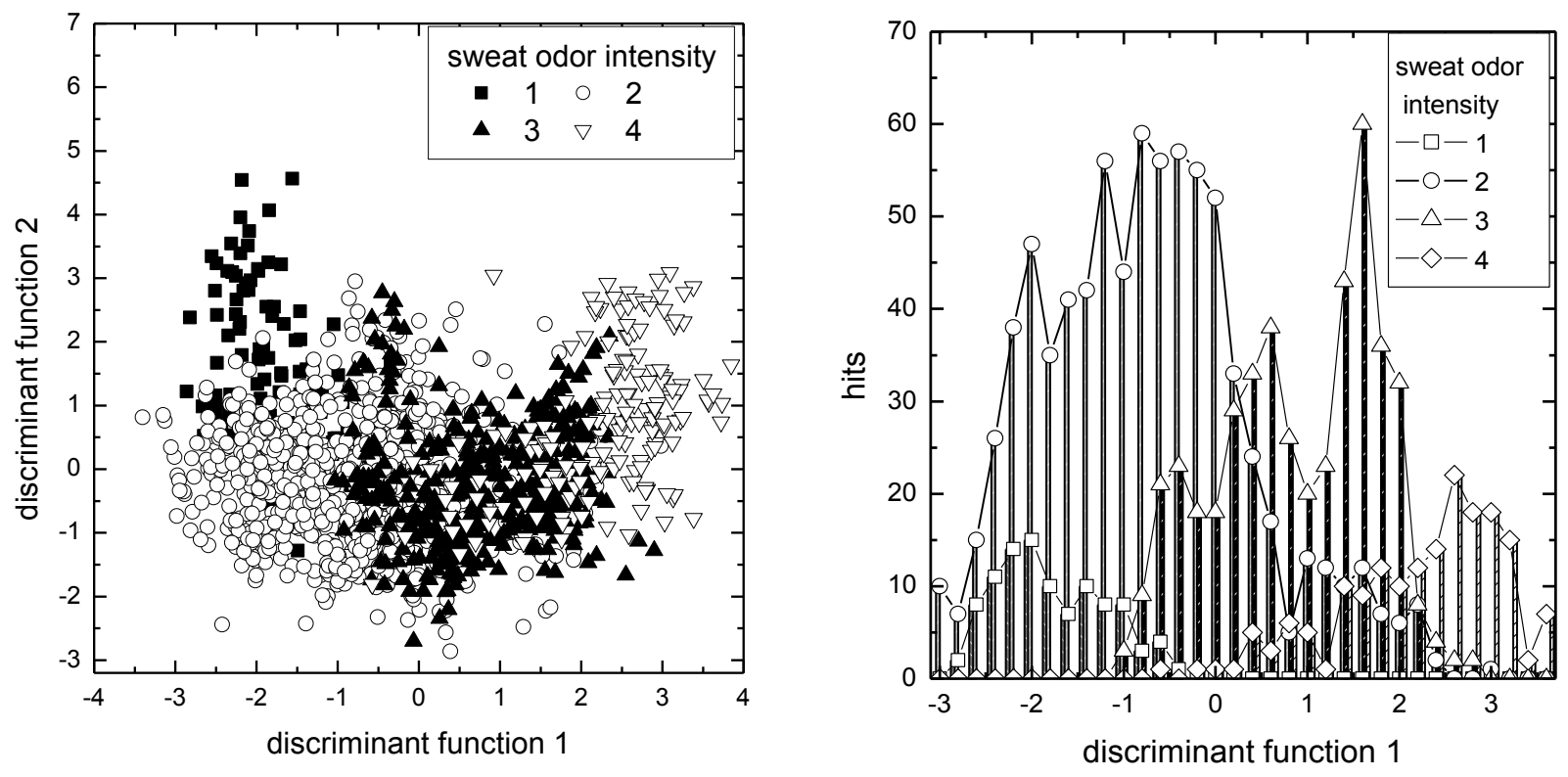

Fig. 5: Left: LDA projection of all tests of shoes worn in daily use at Hohenstein Institutes. The intensity of the sweat odor was evaluated by the human panel on the first, second and seventh day after wearing (total: 96 tests); the parallel measurements of the E-nose system (approx. 30 temperature cycles for each test) were classified according to this intensity on a scale of 1 to 4 (the level 5 was not reached in the tests). The odor intensity shows a good correlation with the first discriminant function (DF1) for the levels 2 to 4 . Odor intensity class 1 and 2 are additionally separated along the second discriminate function (DF2)

Right: the score diagram for DF1 shows nearly Gaussian distribution for all four odor intensity classes.

large overlap between the four classes is evident, but a general trend or correlation between DF1 and the odor score is still evident. The large number of data points results from the approx. $30 \mathrm{~T}$-cycles obtained from each experiment, which are treated as independent data for this projection. However, DF1 would not be sufficient to separate the groups alone, as the groups for odor score 1 and 2 show a strong overlap along DF1, but a slight shift along DF2. When looking at the score distribution of the DF1 values, i.e. number of results with a DF1 value in a certain range, the data show an almost Gaussian distribution for all four groups along DF1, Fig. 5, right. This would indicate a large uncertainty for determining the odor score based on this approach when using the DF1 value to predict the sweat odor intensity. However, one has to take into account that the data themselves are very noisy: the human sensory panel odor values which are used as nominal or reference values for the "training" of the LDA projection have a standard deviation of .4 . In addition, rounding these values to the next full score to separate the data into only four groups means that odor scores which are really very similar (i.e. 2.3 and 2.5) are classified as belonging to groups 2 and 3 , respectively. Therefore, the expectation should not be too high for this approach as the underlying reference itself is not very exact. The problem of adjacent odor scores being inserted in separate classes could be improved by using only data close to the class center value for determining the LDA projection, compare Fig. 4, right.

We have also tested the overall correlation between the human sensory panel and the prediction of a simple artificial neural network (ANN). Only eight relevant features, which were selected based on experience and manual assessment of the patterns, were extracted from the response patterns for the evaluation to prevent overtraining the network. The ANN was a simple feedforward type trained with a standard backpropagation algorithm using a commercial software tool [11]. The odor score obtained from the human sensory panel was used as nominal output for training. Fig. 6 left shows the overall correlation for all temperature cycles of a single sensor over the 96 different experiments with shoes and socks during everyday use. Approx. $2 / 3$ of the data was used for training, the rest as test set to evaluate the ANN performance. It is evident, that for this again simple way of data evaluation and interpretation a good correlation is achieved. Most of the ANN predictions are in the corridor around the ideal output correlation which is defined by the standard deviation of the human test panel assessment.

In further tests with different sensors and more elaborate data evaluation using more features and more sophisticated evaluation techniques, this result should be further improved and tested. In further test series, the system will be tested with completely different shoes and socks. We hope to prove that the system is able to provide objective assessment of the smell of sweat for this specific application. 

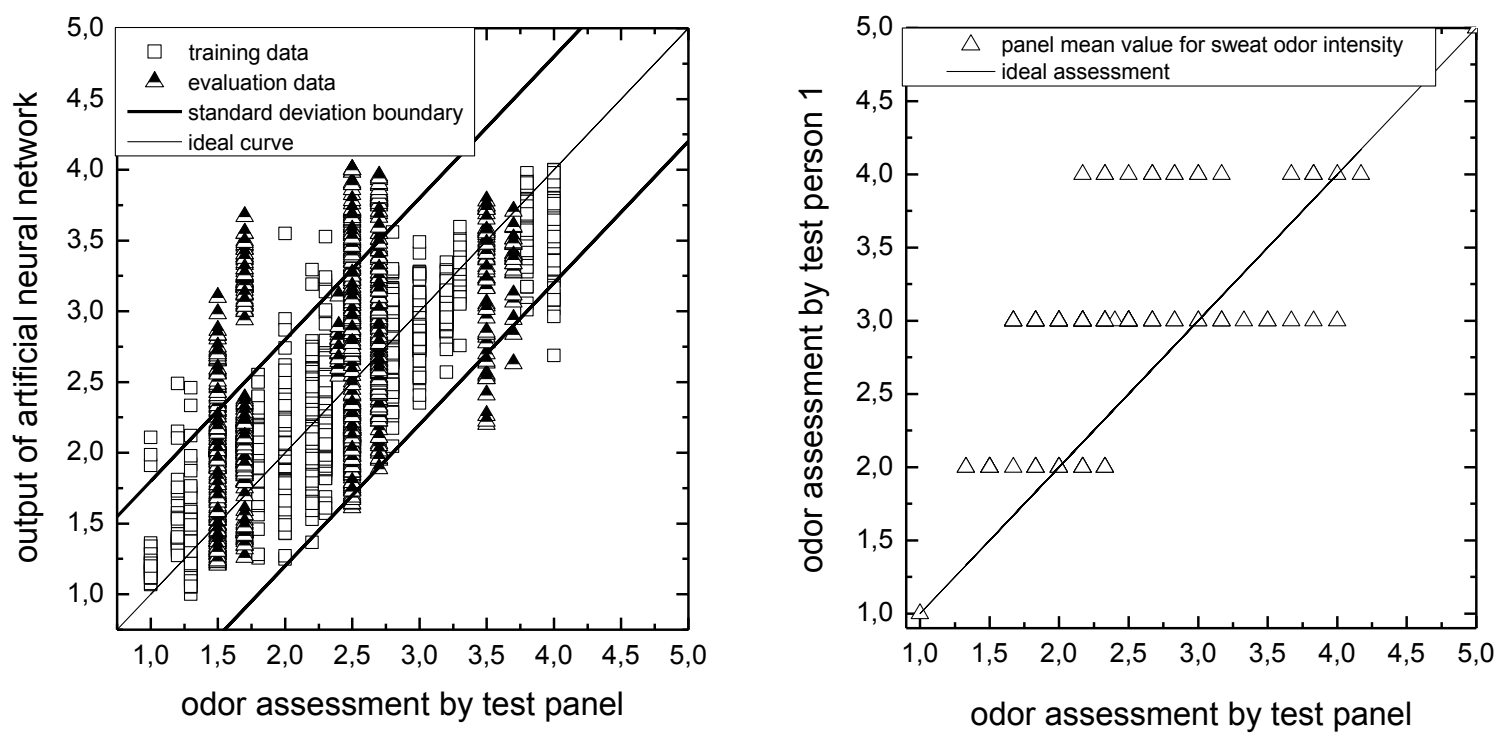

Fig. 6: Left: output of an artifical neural network (ANN) based on the E-nose data of shoes and socks measured on the first, second and seventh day after wearing at Hohenstein Institutes; the network was trained to predict the odor assessment by the human sensory panel. The training data set consisted of all samples with odor scores of the green values show the training data, the blue values are evaluation data; the training data are between 1 and 1.33, between 1.83 and 2.33, between 2.83 and 3.33 and between 3.83 and 4 (higher sores were not obtained). The test data consist of all other samples with odor scores of $1.5,1.66,2.5,2.66,3.5$ and 3.66. A fairly good correlation and prediction of the odor intensity by the ANN is evident. Right: assessment of sweat odor intensity by test person 1 compared to the mean value of the odor assessment by the test panel consisting of 6 test persons; a relatively large variance is evident.

\section{Conclusion}

The hardware of a mobile E-nose system based on a single semiconductor gas sensor was developed for objective evaluation of smell of sweat in shoes and socks. The assessment of a human sensory panel was correllated with the sensor signals obtained from the E-Nose system. For this purpose shoes and socks were sweated by several test persons and assessed afterwards by a human sensory panel as well as measured with the E-nose system. Good correlation within the standard deviation obtained by the sensory panel was achieved both with linear discriminant analysis as with artificial neural networks.

\section{Acknowledgments}

Financial support for this research project $(201 \mathrm{ZN})$ of the research community "Prüf- und Forschungsinstitut Pirmasens e.V." in the program "Industrielle Gemeinschaftsforschung (IGF)", IGF-version ZUTECH, by the Federal Ministry of Economics and Technology via the AiF is gratefully acknowledged.

\section{References}

[1] Bartels, V.T., Umbach, K.H., Schmidt-Fries, U., "Untersuchung der Schweißgeruchsbildung in Textilien mit Hilfe einer elektronischen Nase," Melliand Textilberichte 84(10), (2003), pp. 872-874.

[2] Pearce, T.C., Schiffman, S.S., Nagle, H.T., Gardner, J.W. (eds.), Handbook of Machine Olfaction - Electronic Nose Technology, WILEY-VCH (2003).

[3] Horras, S., Fricke, T., Conrad, T., Engel, M., Schütze, A., "Objective evaluation of smell of sweat for optimal development of shoe/socks systems based on an electronic nose", ISOEN2007, St. Petersburg, Russia.

[4] Gramm, A., Schütze, A., "High performance solvent vapor identification with a two sensor array using temperature cycling and pattern classification," Sensors \& Actuators, B95 (2003), pp. 58-65.

[5] Conrad, T., Hiry, P., Schütze, A., "PuMaH - a temperature control and resistance read-out system for microstructured gas sensors based on PWM signals", Proc. IEEE Sensors Conf., Irvine, CA, Nov. 2005.

[6] UST Umweltsensortechnik $\mathrm{GmbH}$, Geraberg, Germany, http://www.umweltsensortechnik.de

[7] Horras, S., Gaiotto, A., Mayer, M., Reimann, P., Schütze, A., "Correlation of an E-Nose system for odor assessment of shoe/socks systems with a human sensory panel, ISOEN2009, Brescia, Italy, April 15-17, 2009.

[8] Gutierrez-Osuna, R., "Pattern analysis for machine olfaction", IEEE Sensors 2(3) (2002), pp. 189-202.

[9] Schütze, A., Gramm, A., Rühl, T., "Identification of organic solvents by a virtual multisensor system with hierarchical classification," IEEE Sensors Journal, Vol. 4, No. 6 (2004), pp. 857-863.

[10] Lösch, M., Baumbach, M., Schütze, A., "Ozone detection in the ppb-range with improved stability and reduced cross sensitivity", Sensors \& Actuators, B130 (2008), pp. 367-373.

[11] NeuroShell 2.0, Ward Systems Group Inc., USA, http://www.neuroshell.com 